\title{
Summer school in Kabardino- Balkaria by BMSTU SPIE Student Chapter
}

Nikita Chernomyrdin, Kirill Zaytsev, Arsenii Gavdush, Irina Fokina, Valeriy Karasik, et al.

Nikita V. Chernomyrdin, Kirill I. Zaytsev, Arsenii A. Gavdush, Irina N. Fokina, Valeriy E. Karasik, Stanislav O. Yurchenko, "Summer school in KabardinoBalkaria by BMSTU SPIE Student Chapter," Proc. SPIE 9188, Optics Education and Outreach III, 91880Q (15 September 2014); doi: $10.1117 / 12.2061366$

Event: SPIE Optical Engineering + Applications, 2014, San Diego, California, United States 


\title{
Summer school in Kabardino-Balkaria by BMSTU SPIE student chapter
}

\author{
Nikita V. Chernomyrdin, Kirill I. Zaytsev, Arsenii A. Gavdush, Irina N. Fokina, \\ Valeriy E. Karasik, \\ and Stanislav O. Yurchenko \\ Bauman Moscow State Technical University, \\ 105005, 2nd Baumanskaya str. 5/1, Moscow, Russia.
}

\begin{abstract}
This summer BMSTU SPIE Student Chapter have decided to visit Kabardino-Balkaria Republic of Caucasus (Russia) and spent there a week with children in a camp. It was called Summer School. We decided to organize it in order to engage talented and curious children in optics and photonics, and to show them how science could be for fun. Education and entertainment program included such activities as lectures, optical demonstrations, laser games, hiking in the forest, and others. As a result children had a good time outdoors, learned interesting facts and about optics and lasers, and of course found new friends who are keen to know more too. Four Chapter members and about 70 children of age 10-16 took part in this event.
\end{abstract}

Keywords: optical education

\section{IDEA}

Bauman Moscow State Technical University (BMSTU) Student Chapter usually organizes educational activities for students, including lectures, seminars, demonstrations and lab-tours. Often social events are performed for students and children. Having an experience in the school education, we decided to apply it for a big educational activity, which this paper is dedicated for.

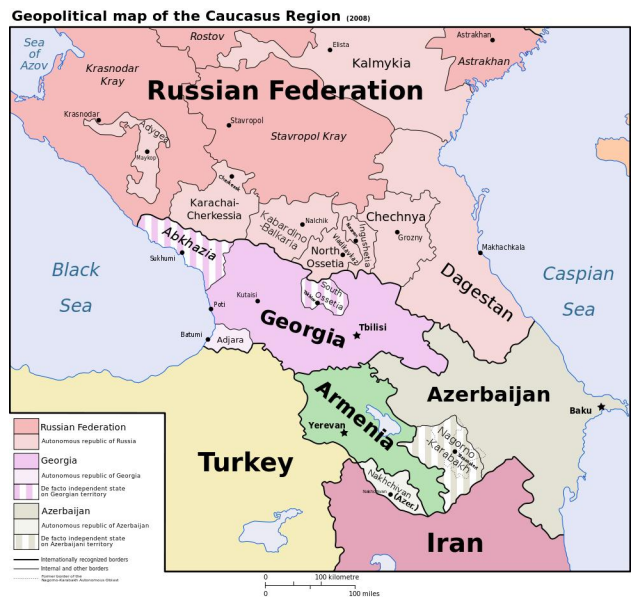

Figure 1. Geopolitical map of the Caucasus Region

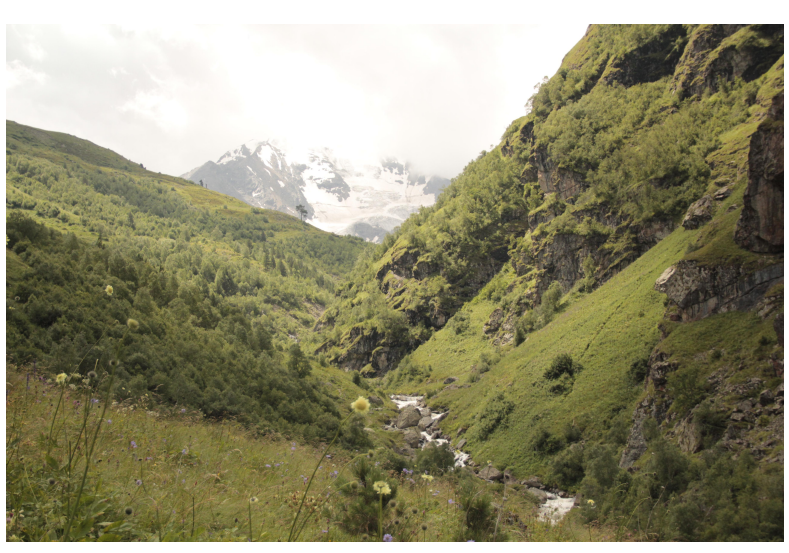

Figure 2. Mountain Cheget

E-mail: chernik-a@yandex.ru 
There are many children who are living far from the big cities like Moscow or St. Petersberg in Russia, and they are interested in science and engineering. Sometimes it is easier to meet a curious child there, than in a big cities, where everything is available. These children have less laboratory and class equipment, less teachers in school, less opportunities to visit museums of science and technical exhibitions. Thus, it is important to help them in self-realization, and to bring something interesting in their educational process. It results in the idea of making some courses for children from remote Russian regions within the summer children camp. Such collaboration was done with the Kabardino-Balkaria Republic of Caucasus (figure $1^{1}$ ). And the educational part, which was organized by BMSTU Chapter, received the name "Summer School".

During this school we tried to speak about optics and photonics within the seminars, games and contests. We have shown children the beauty of the science and how science could be fun, and if you are involved in scientific researches your job will never be boring. We told them a lot about BMSTU and BMSTU SPIE Student Chapter, about possibilities that it provides for students. And in general it was a very good recreation for them, where they breathed the purest air in the mountains (Mountain Cheget (figure 2)).

\section{ACTIVITIES}

In the first day, after we have arrived to the camp, we acquainted with children during some social games. There were 67 children at the age of $10-16$ years.



Figure 3. One of the seminars at the "Summer school"

The first seminar (figure 3) was about Bauman University, our Optical Department and the importance of optics in the daily life. We told them about history of our university, who of the famous scientists and engineers studied there. Also we told them about the present state of education in the university on an example of the Optical Department: what courses and professors a student will find during his graduation there. In this seminar we talked a lot about electronic devices, that all of them are working due to electromagnetic waves, which are essentially the light in different regions of electromagnetic spectrum. We also presented to them our SPIE student chapter, told them about social and professional advantages, when you are the member of the chapter. We told them about organization of lectures and seminars, mutual assistance of all the members in studying and researches, possibilities to take a part in international meetings and conferences. All the seminars were done by the Chapter members. During this time, we tried to make an interesting introduction in the world of optics, 
lasers and photonics with impressive video demonstrations, prepared before in the BMSTU laboratories for this purpose. Also we told about main physical phenomena in optics: reflection, refraction, dispersion, diffraction and polarization. Some theory facts were mixed with experiments. These experiments have made the education process interactive and memorable. In experiment about reflection we use a mirror, protractor under it and a laser. One can visibly detect the angles of incident and reflected laser beams. An experiment that shows Snell's law was prepared using laser and a glass of water. Also in this experiment we have shown them an effect of total internal reflection and offered them to remember, where they could see this effect in the real life in different cases. For example some of them remembered, that this effect they could see when they were swimming and looking inside the water forward and a bit up. Dispersion of light was shown by the classical experiment with a dispersive prism and sunlight. Then we offered them to explain the emergence of the rainbow in the sky by using this knowledge. We have used an effect of birefringence of light in anisotropic medium (calcite crystal) to show the polarization properties of light. To show diffraction we have used a standard experiment with diffraction pattern from a single slit of width equal to wavelength of an incident plane wave from a monochromatic laser source. After lectures and seminars children could apply their new knowledge in practice throughout the optical games.

Moreover, as we all have involved in the scientific work at the University, we prepared lectures about our own research projects, concerning 3D-vision, ${ }^{2}$ medical application of optical devices, ${ }^{3-5}$ photonic crystals ${ }^{6-8}$ spectroscopy and its applications in various cases, ${ }^{9-14}$ and fiber optics. For all of these seminars were explained the main results of the different studies. For example, when we had a seminar about photonic crystals, we have explained its internal structure. These explanation have provided us possibility to explain its main optical properties due to this type of internal structure. Then we have briefly explained the essence of nonlinear optical effects. After that the main result of our research became clear. When we told about spectroscopy, primarily we told about the principles of spectrometers, and for what purposes spectrometry serves. Also we told about problems in processing of spectrometer signals. In this seminar we said a few words about different levels of the molecule or mods, and how they describe the specification of a substance, its physical properties, for example the complex refraction index. After these explanations, the importance of the main results of our researches has been understood by children, and they have seen the main direction of the future researches. It has led to an interest and desire to join our researches in the near future.

We showed numerical simulations of the light propagation in different media using FDTD modeling software, instructions how to make our own hologram, and explain the operation of microscope, telescope, CCD, etc. Of course, they have not been prepared to understand all the details, primarily in mathematics, but the main physics goals of our researches have become understandable such as their necessity and urgency.

The funniest event of these lectures was connected with optical games, which partly were developed by the members of SPIE, SPIE student chapters. ${ }^{15,16}$ These games, such as Hit the target ${ }^{16}$ and Laser Chess, ${ }^{17,18}$ helped to communicate with children in the less formal atmosphere (figure 4). These games developed creative scientific manner of thinking in children, because one must have considerable ingenuity to achieve a good result in such games we provided.

Here are some details of these games. For example, this is a description how the game "Hit the target" was organized in this summer school. Children were divided into a number of teams. Every team has its own table with some optical components: moveable mirrors, stable optical obstacle, lenses and diffraction grating for the last round of the game. The obstacle was a glass with water inside. Also all commands had several protractors to measure the angles, and a ruler. There was a task to hit their target, printed on paper, with a laser beam. Laser position was stable and it was turned off during the preparing. The preparing time was used to expose mirrors to the appropriate positions measuring the angles of reflection. There were four rounds in this game. The first round required only one mirror in the optical system between the laser and the target, and they were in one plane. The next round was for two mirrors in system, the third round required three mirrors and laser, which was not in the same plane as target. In the fourth round children had to pay attention to the glass of water and its refraction properties. The last round was the most difficult one: in the end of an optical system they must put lenses and a grating between them, so that laser beam will be collimated to the target. In case we had described all main physical phenomena in optics including reflection, refraction and diffraction before the game, they came prepared for it. Between the teams it was a competition: which team will hit the target 


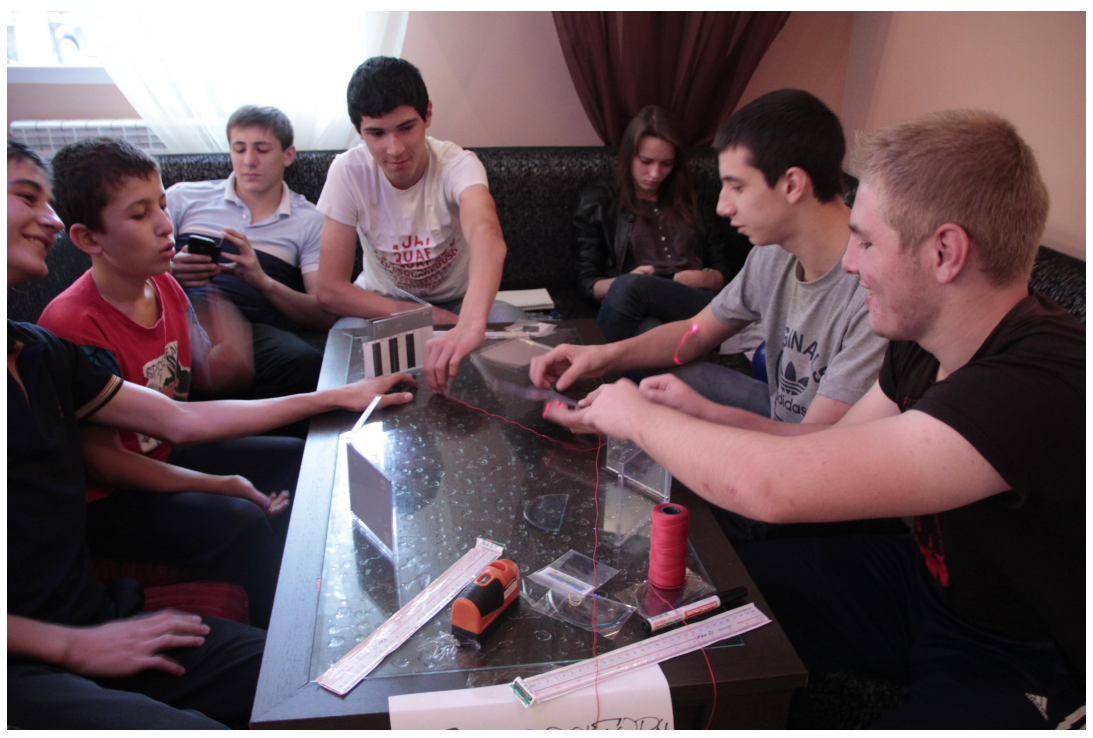

Figure 4. During the game Hit the target

first and how accurate this hit will be. All commands had to describe, how their optical system works and which optical phenomena they used in their optical solution. The command, that won the game got an optical kit with the stuff including laser pointer, mirrors, and also some lenses and diffraction gratings. This kit was given to them for the future optical experiments.

When children were playing Laser Chess it was a competition too, between all players. The most lucky and intelligent player got a box with chess as a prize after the game.

In addition to scientific studies, children had time for social activities with each other and us, their teachers. At first, we had the so-called wild day when one should become a native man or woman, make a tribe, create a motto, dance and song. Everybody voted for the tribe and the best tribe was chosen (see the photo, figure 5).

Also we had the hiking in the forest. There we had a time to sit near the fire, where we singing and talking about future plans. It was excellent to hear, that most of these children started to think about BMSTU as their future University. It is a good way to find friends and share experience in science with children (figure 6).

One day we spent in the Caucasus Mountains. It is a very beautiful place with unique nature, mountain rivers, waterfalls, and we visited it all together. Whole day we were walking and climbing there. It was the best way for recreation for all of us and great opportunity for the children to know more about their homeland.

It is rather important to support talented children. For this purpose we have told them about educational programs and contests for children in BMSTU. This information will help them to realize and improve their skills and knowledge, because there is an opportunity to start your our research project at school and make it under the guidance of the master/PhD students or professors from the BMSTU. Such collaboration is available online.

For our Chapter this event became the first and the longest educational event outside Moscow region. And we are proud of our success. We have tried to teach them all that we know in optical science and learned many of the subtleties of child psychology, which must be considered when planning lessons. We understand that we made something important for children from one of the remote regions, trying to make this time unforgettable for them. Meanwhile, this time became unforgettable for us.

This event was inspired by the SPIE Student Chapters all over the world and SPIE educational activities. 


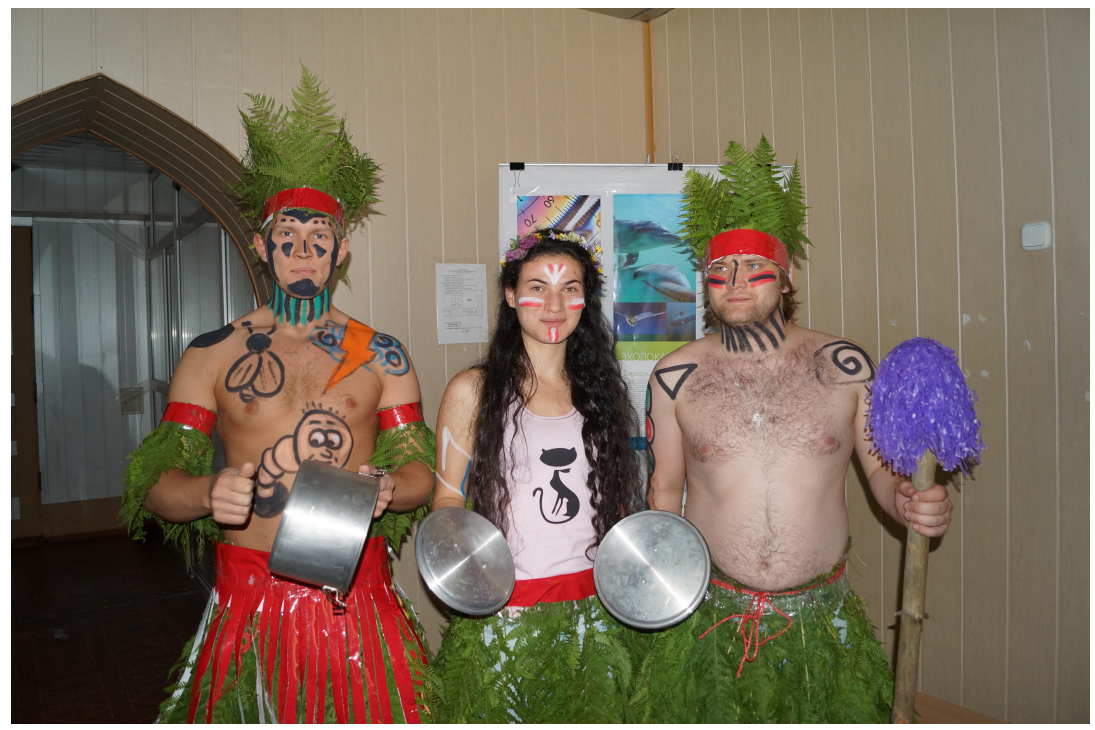

Figure 5. The best tribe

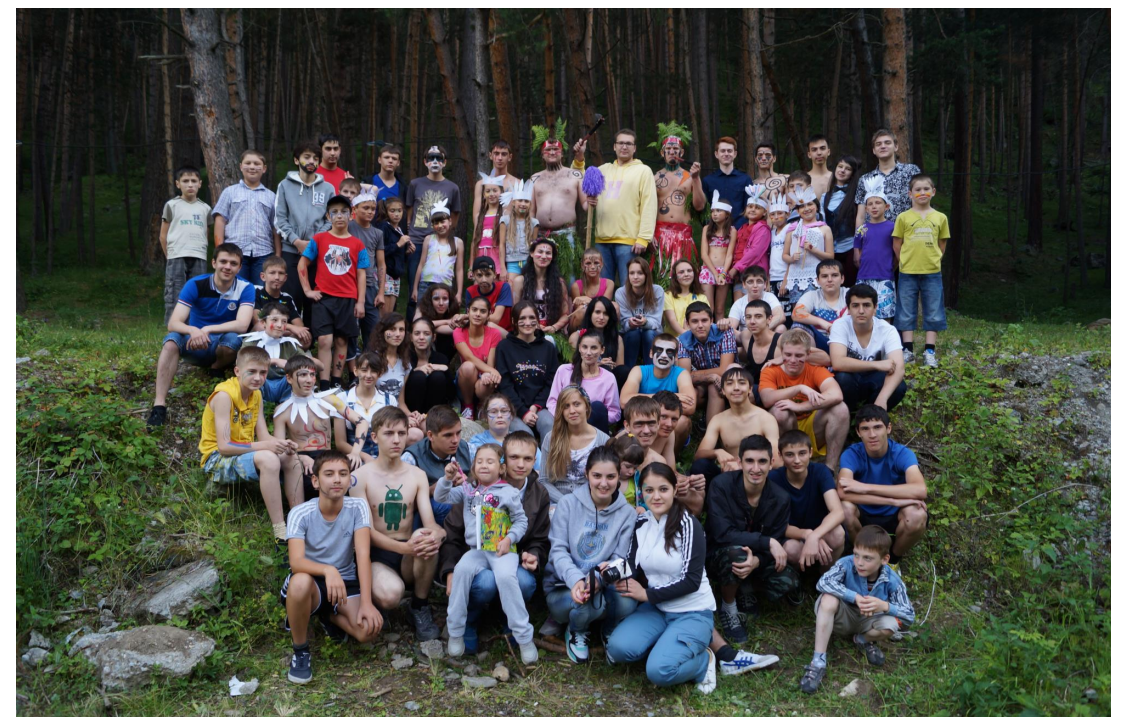

Figure 6. Hiking in the forest

\section{REFERENCES}

[1] From Wikipedia, t. f. e., "Caucasus," (July 2014).

[2] Zaytsev, K. I., Perov, A. N., Fokina, I. N., Karasik, V. E., Yakovlev, E. V., and Yurchenko, S. O., "Bwo based thz imaging system," Journal of Physics: Conference Series 486, 012027 (2014).

[3] Zaytsev, K. I., Kudrin, K. G., Koroleva, S. A., Fokina, I. N., Volodarskaya, S. I., Novitskaya, E. V., A. N, P., Karasik, V. E., and Yurchenko, S. O., "Medical diagnostics using terahertz pulsed spectroscopy," Journal of Physics: Conference Series 486, 012014 (2014). 
[4] Volodarskaya, S. I., Zaytsev, K. I., Karasik, V. E., Novitskaya, E. V., and Fokina, I. N., "Experimental stadying the ability of tooth enamel early cariosity diagnosis with terahertz spectroscopy," Herald of Bauman Moscow State Technical University. Series: "Instrumentation" 2, 33-47 (2013).

[5] Zaytsev, K. I., Karasik, V. E. Koroleva, S. A., and Fokina, I. N., "Study the ability of terahertz imaging systems application for medical diagnosis," Herald of Bauman Moscow State Technical University. Series: "Instrumentation" 4, 114-127 (2012).

[6] Zaytsev, K. I., Katyba, G. M., Yakovlev, E. V., Gorelik, V. S., and Yurchenko, S. O., "Band-gap nonlinear optical generation: The structure of internal optical field and the structural light focusing," Journal of Applied Physics 115(21) (2014).

[7] Zaytsev, K. I. and Yurchenko, S. O., "Enhancement of second harmonic generation in nano2-infiltrated opal photonic crystal using structural light focusing," Applied Physics Letters 105(5) (2014).

[8] Zaytsev, K. I., Gorelik, V. S., Khorokhorov, A. M., and Yurchenko, S. O., "Fdtd simulation of the electromagnetic field surface states in 2d photonic crystals," Journal of Physics: Conference Series 486, 012003 (2014).

[9] Zaytsev, K. I., Chernomyrdin, N. V., Gorevoy, A. V., Trofimov, N. E., Fokina, I. N., Alekhnovich, V. I., Karasik, V. E., and Yurchenko, S. O., "An approach for automatic construction of the wavelet-domain denoising procedure for thz pulsed spectroscopy signal processing," Journal of Physics: Conference Series $\mathbf{4 8 6}$, 012034 (2014).

[10] Zaytsev, K. I., Fokina, I. N., Fedorov, A. K., and Yurchenko, S. O., "Sensing of phase transition in medium with terahertz pulsed spectroscopy," Journal of Physics: Conference Series 486, 012024 (2014).

[11] Zaytsev, K. I., Gavdush, A. A., Karasik, V. E., Alekhnovich, V. I., Nosov, P. A., Lazarev, V. A., Reshetov, I. V., and Yurchenko, S. O., "Accuracy of sample material parameters reconstruction with terahertz pulsed spectroscopy," Journal of Applied Physics 115(20) (2014).

[12] Yakovlev, E. V., Zaytsev, K. I., Fokina, I. N., Karasik, V. E., and Yurchenko, S. O., "Nondestructive testing of polymer composite materials using thz radiation," Journal of Physics: Conference Series 486, 012008 (2014).

[13] Zaytsev, K. I., Chernomyrdin, N. V., and Alekhnovich, V. I., "Novel technique for medium permittivity profile reconstruction using thz pulsed spectroscopy," Journal of Physics: Conference Series 486, 012010 (2014).

[14] Zaytsev, K. I., Karasik, V. E., Fokina, I. N., and Alekhnovich, V. I., "Invariant embedding technique for medium permittivity profile reconstruction using terahertz time-domain spectroscopy," Optical Engineering 52(6), 068203 (2013).

[15] Haist, T. and Burla, A., "Learning by playing: how to create the perfect learning game for and with optics," Proceedings of SPIE $\mathbf{7 7 8 3}(0 \mathrm{E}), 10$ (2010).

[16] Chapter, U. L. S. S., "The 2010 photonic games: Learning optics while having fun," (2010).

[17] Wilk, S. R., "Playing with light: A history of games that incorporate the photon," Optics and Photonics News (Optical Society of America) 18(10), 18 (2007).

[18] Duppong, M., "Laser chess," Compute! , 25 (1987). 